\title{
Fibroblast growth factor 21 inhibition aggravates cardiac dysfunction in diabetic cardiomyopathy by improving lipid accumulation
}

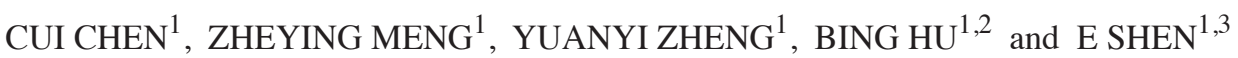 \\ ${ }^{1}$ Department of Ultrasound in Medicine; ${ }^{2}$ Shanghai Institute of Ultrasound in Medicine, Shanghai Jiao Tong University \\ Affiliated Sixth People's Hospital, Shanghai 200233; ${ }^{3}$ Department of Ultrasound in Medicine, Tong Ren Hospital, \\ Shanghai Jiao Tong University School of Medicine, Shanghai 200336, P.R. China
}

Received January 31, 2017; Accepted July 27, 2017

DOI: 10.3892/etm.2017.5375

\begin{abstract}
Diabetic cardiomyopathy (DCM) is one of the major causes of morbidity and mortality in diabetic patients. Recent studies have demonstrated an increased level of fibroblast growth factor 21 (FGF21) in the plasma of DCM patients, and FGF21 has been proven to be a cardiovascular protector of the heart. The present study aimed to further investigate the pathogenic role of FGF21 in DCM, hypothesizing that a lack of FGF21 may promote the progression of DCM by regulating the lipid metabolism, cardiac hypertrophy and cardiac fibrosis, thus deteriorating the cardiac dysfunction. A total of 44 mice were randomly assigned into the normal $(n=6)$, DCM $(n=6)$, normal + scrambled siRNA $(n=6), D C M+$ scrambled siRNA $(n=6)$, normal + FGF21 siRNA $(n=10)$ and DCM + FGF21 siRNA $(n=10)$ groups. Type 1 diabetes mellitus was induced to mice in the DCM groups by streptozotocin injection, while FGF21 expression was inhibited by FGF21 siRNA. Normal and DCM mice administrated with scrambled siRNA were respectively regarded as the controls for the normal + FGF21 siRNA and DCM + FGF21 siRNA groups. In the DCM group, FGF21 inhibition promoted cardiac hypertrophy and fibrosis, and the expression levels of their indicators, including atrial natriuretic factor, $\alpha$-skeletal actin, collagen type I and III, and transforming growth factor- $\beta$, increased, leading to further decreased cardiac function. In addition, FGF21 inhibition in DCM mice elevated the quantity of lipid droplets and the concentration of heart triglycerides, plasma triglycerides and cholesterol levels, accompanied by downregulation of peroxisome proliferator-activated receptor $\gamma$ co-activator $1 \alpha(\mathrm{PGC}-1 \alpha)$ and upregulation of cluster of differentiation
\end{abstract}

Correspondence to: Professor E Shen, Department of Ultrasound in Medicine, Shanghai Jiao Tong University Affiliated Sixth People's Hospital, 600 Yishan Road, Shanghai 200233, P.R. China

E-mail: shene126@126.com

Key words: fibroblast growth factor 21, diabetic cardiomyopathy, cardiac hypertrophy, lipid accumulation
(CD)36. Thus, the results indicated that FGF21 inhibition exacerbates the cardiac dysfunction by aggravating the lipid accumulation through regulating the expression levels of PGC-1 $\alpha$ and CD36. In conclusion, it is suggested that FGF21 may be a potentially useful agent in the treatment of DCM.

\section{Introduction}

Diabetic cardiomyopathy (DCM) serves an essential role in the progression of diabetes, and is one of the major causes of morbidity and mortality in patients with diabetes. DCM prevalence is $\sim 12 \%$ in the population of patients with diabetes, and causes heart failure and mortality (1). It has been reported that intracellular lipid accumulation, altered cell signaling, high glucose-induced generation of advanced glycation end products and reactive oxygen species, and altered fuel use in the hearts of patients with diabetes are considered as risk factors for DCM (2). Fibroblast growth factor 21 (FGF21) is a secreted protein involved in the regulation of the glucolipid metabolism, ketogenesis and insulin sensitivity $(3,4)$. A previous study has detected an increased level of FGF21 in the plasma of DCM patients (5).

Studies have attempted to investigate the association between FGF21 and cardiac function, and observed that the heart was a target and also a source of FGF21, the heart expresses and releases FGF21, which protects against cardiac hypertrophy (6). Elevated FGF21 levels have been identified in patients with carotid atherosclerosis, hypertension and coronary artery disease (7-9). Furthermore, FGF21 has been observed to serve a role as an antioxidant factor, preventing pro-oxidative pathways, which were induced by inflammatory or hypertrophic conditions (10). FGF21 may also be involved in the development and maintenance of atrial fibrosis in atrial fibrillation accompanied with rheumatic heart disease (11). In Chinese patients, the serum FGF21 level is independently associated with the presence of acute myocardial infarction. In addition, high FGF21 levels may be associated with the incidence of re-infarction within 30 days of the first myocardial infarction (12).

These aforementioned findings suggest that FGF21 may be a critical metabolic hormone with an important function in the 
cardiovascular system. However, the mechanism underlying the function of this metabolic hormone in DCM remains unclear; thus, investigating the role of FGF21 in DCM is of considerable interest. The present study investigated the hypothesis that FGF21 may affect the progression of DCM by regulating the lipid metabolism, myocardial hypertrophy and fibrosis. The metabolic status, cardiac structure and function of normal and DCM mice treated with or without FGF21 siRNA for inhibition of FGF21 levels were examined.

\section{Materials and methods}

Animal models. The animal experiments were conducted in accordance with the guidelines of the National Institutes of Health of China for the care and use of laboratory animals. Experimental protocols were approved by the local Animal Ethics Committee affiliated to the Shanghai Sixth People's Hospital (Shanghai, China). A total of 44 male C57/BL6J mice were provided by the Animal Laboratory of the Shanghai Sixth People's Hospital. All mice were housed in housing units with a $12 \mathrm{~h}$ light/dark cycle and these units were maintained at $25^{\circ} \mathrm{C}$ with $\sim 50 \%$ relative humidity. The mice received a normal diet and water ad libitum.

The mice were randomly assigned into the normal $(n=6)$, DCM $(n=6)$, normal + scrambled siRNA $(n=6)$, DCM + scrambled siRNA $(n=6)$, normal + FGF21 siRNA $(n=10)$ and DCM + FGF21 siRNA $(n=10)$ groups. Type 1 diabetes mellitus was induced to mice in the DCM, DCM + scrambled siRNA and DCM + FGF21 siRNA groups by intraperitoneal (IP) injection of streptozotocin (STZ; Sigma-Aldrich; Merck, Darmstadt, Germany) at the dose of $150 \mathrm{mg} / \mathrm{kg}$. Saline was administered by IP injection to the mice in the normal, normal + scrambled siRNA and normal + FGF21siRNA groups. After $72 \mathrm{~h}$, the Type 1 diabetes mellitus mouse model was successfully established, with mice exhibiting blood glucose levels of $>16.9 \mathrm{mmol} / 1$. At week 12 after the DCM model was established, FGF21 siRNA and scrambled siRNA (Shanghai GenePharma Co., Ltd., Shanghai, China) were dissolved in a diethyl pyrocarbonate and glucose solution at $10 \mu \mathrm{g} / \mu \mathrm{l}$, and administrated by injection through the tail vein at a dose of $5 \mathrm{mg} / \mathrm{kg}$ in mice of normal + FGF21 siRNA, DCM + FGF21 siRNA, normal + scrambled siRNA, DCM + scrambled siRNA groups. The siRNA sequences were as follows: FGF21 siRNA, 5'-CCAACAACCAGAUGGAACUTT-3' (sense) and 5'-TTGGUUGUUGGUCUACCUUGA-3' (antisense); scrambled siRNA, 5'-UUCUCCGAACGUGUCACGUTT-3' (sense) and 5'-ACGUAGCACGUUCGGAGAATT-3' (antisense). In the siRNA absent groups (the normal and DCM groups) saline was administrated to the mice by intravenous injection through the tail vein. In mice of the normal + FGF21 siRNA and the DCM + FGF21 siRNA groups, FGF21 expression was inhibited by FGF21 siRNA injection.

Transthoracic echocardiography (TTE) was performed 1 week after injection to evaluate the cardiac function. Blood samples were collected and stored at $4^{\circ} \mathrm{C}$ prior to TTE; following TTE the mice were sacrifice and their hearts and livers were extracted. The tissues used for hematoxylin and eosin (H\&E) and Masson's staining, and biochemical tests were stored in liquid nitrogen. Tissues used for transmission electron microscope (TEM) analysis were fixed in $2.5 \%$ glutaraldehyde overnight at $4^{\circ} \mathrm{C}$. The heart and liver samples were used to examine the FGF21 expression levels, cardiac morphologic and fibrotic changes, condition of lipid accumulation and concentration of peroxisome proliferator-activated receptor $\gamma$ co-activator $1 \alpha$ (PGC-1 $\alpha)$ and cluster of differentiation (CD)36. Differences in the acquired parameters among the groups were compared to evaluate the cardiac function and structure, degree of myocardial hypertrophy and fibrosis, and the cardiac metabolism state, as well as to deduce the possible mechanism behind these differences. These results would assist in clarifying the possible mechanism underlying the effect of FGF21 on the progression of DCM.

Echocardiography. TTE was performed to evaluate the cardiac function and structure using the VisualSonics Vevo 2100 high-resolution imaging system (FUJIFILM VisualSonics, Inc., Toronto, ON, Canada). Each mouse was placed in a supine position following anesthesia by $1.5 \%$ sodium pentobarbital (Tianjin Jinyao Amino Acid Co., Ltd., Tianjin, China) at the dose of $75 \mathrm{mg} / \mathrm{kg}$ (intraperitoneal injection). A heating pad was used to warm the body. Subsequently, two-dimensional TTE and M-mode TTE were applied to acquire the parameters that indicate the cardiac structure and function, including ejection fraction (EF), fractional shortening (FS), left ventricular (LV) mass, interventricular septum during diastole (IVSd), interventricular septum during systole (IVSs), LV internal diameter during diastole (LVIDd), LV internal diameter during systole (LVIDs), posterior wall thickness during diastole (PWTd) and posterior wall thickness during systole (PWTs). These indicators were measured three times and the mean value was used in statistical analysis.

Plasma FGF21 level assessment. In order to observe the effectiveness of FGF21 siRNA injection, the plasma FGF21 levels were measured. Briefly, 1 week after siRNA injection, whole blood was collected from the mice and centrifuged at $1,509 \mathrm{xg}$ for $10 \mathrm{~min}$ at $4^{\circ} \mathrm{C}$. The plasma was then collected and the FGF21 concentration was measured by a FGF21 Quantikine ELISA kit (cat. no. MF2100; R\&D systems, Inc., Minneapolis, MN, USA), according to the manufacturer's protocol.

\section{Reverse transcription-quantitative polymerase chain reaction} $(R T-q P C R)$. RT-qPCR was used to measure the expression levels of cardiac and liver FGF21 mRNA. In addition, the levels of indicators of myocardial hypertrophy and fibrosis were determined, including atrial natriuretic factor (ANF), $\alpha$-skeletal actin ( $\alpha$-SKA), collagen type I (Col I), Col III and transforming growth factor- $\beta$ (TGF- $\beta$ ). Total RNA was extracted by TRIzol reagent (Takara Bio, Inc., Shiga, Japan) according to the manufacturer's instructions. A total of $500 \mathrm{ng}$ RNA and the PrimeScript II 1st Strand cDNA Synthesis Kit (cat. no. 6210A; Takara Bio, Inc.) were used for cDNA synthesis. PrimeScript ${ }^{\mathrm{TM}}$ RT Reagent Kit (Perfect Real Time; cat. no. RR037A; Takara, Bio, Inc.) and a 7500 HT Fast Real-Time PCR System (Applied Biosystems; Thermo Fisher Scientific, Inc., Waltham, MA, USA) were used to perform a qPCR analysis. For the qPCR analysis, Universal SYBR Green I (Bioteke Corporation, Beijing, China) was used. The initial denaturation was $95^{\circ} \mathrm{C}$ for $30 \mathrm{sec}, 40$ cycles of denaturation at $95^{\circ} \mathrm{C}$ for $5 \mathrm{sec}$, annealing at $60^{\circ} \mathrm{C}$ for $30 \mathrm{sec}$ and elongation at 
Table I. Primer sequences for quantitative polymerase chain reaction.

\begin{tabular}{lll}
\hline Gene & \multicolumn{1}{c}{ Forward primer $\left(5^{\prime}-3^{\prime}\right)$} & \multicolumn{1}{c}{ Reverse primer $\left(5^{\prime}-3^{\prime}\right)$} \\
\hline ANF & GAGCAGACCGATGAAGCG & AGTGGCAATGCGACCAAG \\
$\alpha-$ SKA & ATCTCACGTTCAGCTGTGGTCA & ACCACCGGCATCGTGTTGGAT \\
TGF- $\beta$ & TGCGCCTGCAGAGATTCAAG & AGGTAACGCCAGGAATTGTTGCTA \\
Col I & GACGCATGGCCAAGAAGACA & GCACCAGGAGGACCAGGAAGT \\
Col III & AAACTGGTGAAGGTGGCTATG & TTTTCACCTCCAACTCCAATG \\
FGF2 1 & GGTGCTGCCAAGGCTGTGGG & CCAGGCGGCATGTCAGATCCAC \\
GAPDH & AATGGATTTGGACGCATTGGT & TTTGCACTGGTACGTGTTGAT
\end{tabular}

ANF, atrial natriuretic peptide; $\alpha$-SKA, $\alpha$-skeletal actin; TGF- $\beta$, transforming growth factor- $\beta$; Col, collagen; FGF21, fibroblast growth factor 21 .

$72^{\circ} \mathrm{C}$ for $30 \mathrm{sec}$, and a final elongation at $72^{\circ} \mathrm{C}$ for $10 \mathrm{~min}$. Values were normalized by levels of GAPDH mRNA and presented as an expression fold change using the $2^{-\Delta \Delta \mathrm{Cq}}$ method (13). The primers were synthesized by Shanghai GenePharma Co., Ltd. The primers used in qPCR are listed in Table I.

$H \& E$ and Masson's trichrome staining. H\&E and Masson's trichrome staining were used to compare the histology of the liver and myocardial tissue in different groups. Briefly, subsequent to harvesting, the tissues were fixed in $10 \%$ neutral-buffered paraformaldehyde, embedded in paraffin and sliced into 4- $\mu$ m-thick sections. Deparaffinization of the section was then performed, followed by staining with $\mathrm{H} \& \mathrm{E}$ to determine the morphology of the cardiomyocytes and liver tissues, while Masson's trichrome staining was applied in order to assess the degree of myocardial fibrosis. The staining was examined under a light microscope. The cardiomyocyte areas were assessed by ImageJ software (version 1.44; National Institutes of Health, Bethesda, MD, USA).

Measurements of cardiac lipid droplets under a TEM. Counting of lipid droplets was applied to evaluate the level of cardiac metabolism. Briefly, following sectioning into cubes of $\sim 1 \mathrm{~mm}^{3}$ in size, the myocardial tissues were fixed with $2.5 \%$ glutaraldehyde overnight at $4^{\circ} \mathrm{C}$, then washed three times with $0.1 \mathrm{~mol} / 1$ phosphate-buffered saline $(\mathrm{pH}=7.4)$. Subsequently, the samples were fixed in $1 \%$ osmium tetroxide for $2 \mathrm{~h}$ at $4^{\circ} \mathrm{C}$, washed three times by phosphate buffered solution, dehydrated in a graded ethanol series and embedded in epoxy resin. The cubes were then stained with uranyl acetate and lead citrate, cut into ultrathin sections $(80 \mathrm{~nm})$ with a diamond knife and collected on 300-mesh copper or nickel grids. Next, the samples were examined under a TEM to count the cardiac lipid droplets.

Metabolic parameter assessment. The heart tissues were homogenized in the standard diluted assay reagent $(10 \mu \mathrm{l} / \mathrm{mg}$ tissue) and then centrifuged at $10,000 \mathrm{x} \mathrm{g}$ for $10 \mathrm{~min}$ at $37^{\circ} \mathrm{C}$. The resultant supernatant was then used to measure the triglyceride concentration with a Triglyceride Colorimetric Assay kit (Cayman Chemicals, Ann Arbor, MI, USA). Plasma triglyceride and cholesterol levels were also measured by colorimetric methods using a triglyceride E-test and a cholesterol E-test (Wako Pure Chemical Industries, Ltd., Japan), respectively.

Western blot analysis. Western blot analysis was used to measure the myocardial concentrations of PGC-1 $\alpha$ and CD36. Briefly, total protein was separated by $10 \%$ SDS-PAGE and then transferred to a nitrocellulose membrane, which was blocked by $5 \%$ skimmed milk powder solution for $1 \mathrm{~h}$. Next, the protein membrane was incubated overnight at $4^{\circ} \mathrm{C}$ with the primary antibodies, including anti-PGC-1 $\alpha$ (cat no. ab54481; 1:1,000), anti-CD36 (cat no. ab133625; 1;2,000) and the internal antibody, anti-GAPDH (cat. no. ab181602; 1:10,000) (all Abcam, Cambridge, MA, USA). Any unbound antibodies in the membrane were washed using $0.1 \%$ Tween 20 in tris-buffered saline, and then the membrane was incubated with horseradish peroxidase-conjugated goat anti-rabbit antibodies (cat. no. ab205718; 1:2,000; Abcam) for $1 \mathrm{~h}$ at room temperature. Subsequently, the antigen-antibody complex was detected with an enhanced chemiluminescence detection kit (EMD Millipore, Billerica, MA, USA). The grey levels of blots were quantified by ImageJ software (version 1.44).

Statistical analysis. SPSS statistical software (version 21; IBM Corp., Armonk, NY, USA) was used to conduct all the data analysis. The data are expressed as the mean \pm standard deviation in all groups, and differences among the results were determined by one-way analysis of variance and post hoc Turkey's test. $\mathrm{P}<0.05$ was considered as indication of a statistically significant difference.

\section{Results}

Analyses of body weight and blood glucose. The blood glucose levels of mice in the DCM, DCM + scrambled siRNA and $\mathrm{DCM}+\mathrm{FGF} 21 \mathrm{siRNA}$ groups increased to $>16.9 \mathrm{mmol} / \mathrm{l}$ at $72 \mathrm{~h}$ after STZ injection (Table II). Significant differences $(\mathrm{P}<0.05)$ were identified between the mice injected with STZ and non-treated mice in the normal, normal + scrambled siRNA and normal + FGF21 siRNA groups, while no significant difference existed between these three groups, indicating that the diabetes model was successfully established. Prior to sacrifice, the body weights of mice in the three DCM groups were significantly lower compared with those in the three normal groups $(\mathrm{P}<0.05)$. 
Table II. Alterations in body weight and blood glucose in mice.

$72 \mathrm{~h}$ after STZ injection

\begin{tabular}{|c|c|c|c|c|}
\hline \multirow[b]{2}{*}{ Group } & & \\
\hline & Body weight (g) & Blood glucose (mmol/l) & Body weight (g) & Blood glucose (mmo \\
\hline $\mathrm{N}$ & $23.55 \pm 1.21$ & $8.4 \pm 1.68$ & $29.4 \pm 1.59$ & $9.75 \pm 1.84$ \\
\hline $\mathrm{N}+\mathrm{Scsi}$ & $23.37 \pm 1.75$ & $8.9 \pm 1.20$ & $29.2 \pm 1.37$ & $10.15 \pm 1.24$ \\
\hline $\mathrm{N}+\mathrm{FGF} 21 \mathrm{si}$ & $23.26 \pm 1.47$ & $9.63 \pm 0.67$ & $29.09 \pm 1.46$ & $10.48 \pm 0.94$ \\
\hline DCM & $23.73 \pm 2.05$ & $20.93 \pm 2.03^{\mathrm{a}}$ & $22 \pm 1.16^{\mathrm{a}}$ & $24.21 \pm 2.65^{\mathrm{a}}$ \\
\hline $\mathrm{DCM}+\mathrm{Scsi}$ & $22.98 \pm 1.12$ & $21.67 \pm 1.98$ & $21.83 \pm 1.2$ & $23.9 \pm 2.18$ \\
\hline $\mathrm{DCM}+\mathrm{FGF} 21 \mathrm{si}$ & $23.11 \pm 1.85$ & $21.84 \pm 2.11^{\mathrm{a}}$ & $21.54 \pm 0.96^{\mathrm{a}}$ & $29.79 \pm 4.48^{b}$ \\
\hline
\end{tabular}

${ }^{a} \mathrm{P}<0.05$ vs. N group; ${ }^{\mathrm{b}} \mathrm{P}<0.05$ vs. DCM group. Data were analyzed by one-way analysis of variance, followed by a post hoc Turkey's test. Values are reported as the mean \pm standard deviation. N, normal; DCM, diabetic cardiomyopathy; Scsi, scrambled siRNA; FGF21si, FGF21 siRNA; FGF21, fibroblast growth factor 21; STZ, streptozotocin.

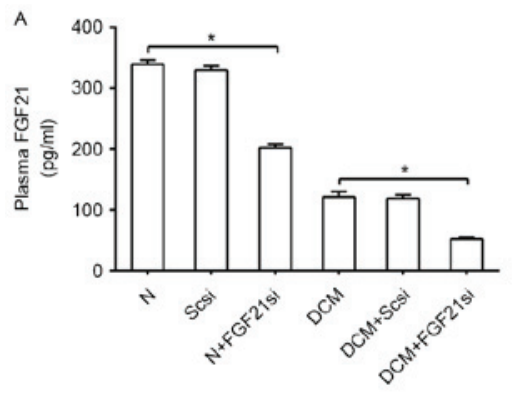

D

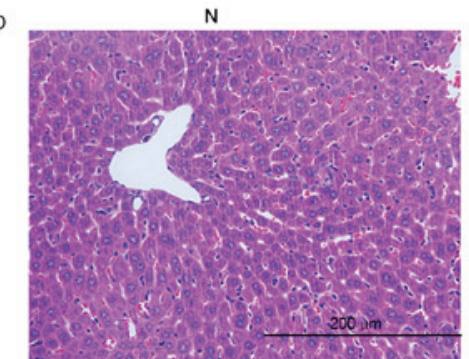

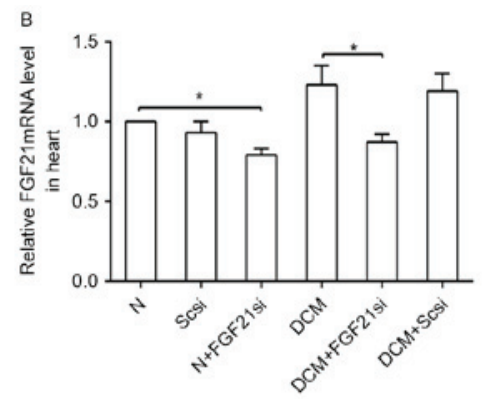
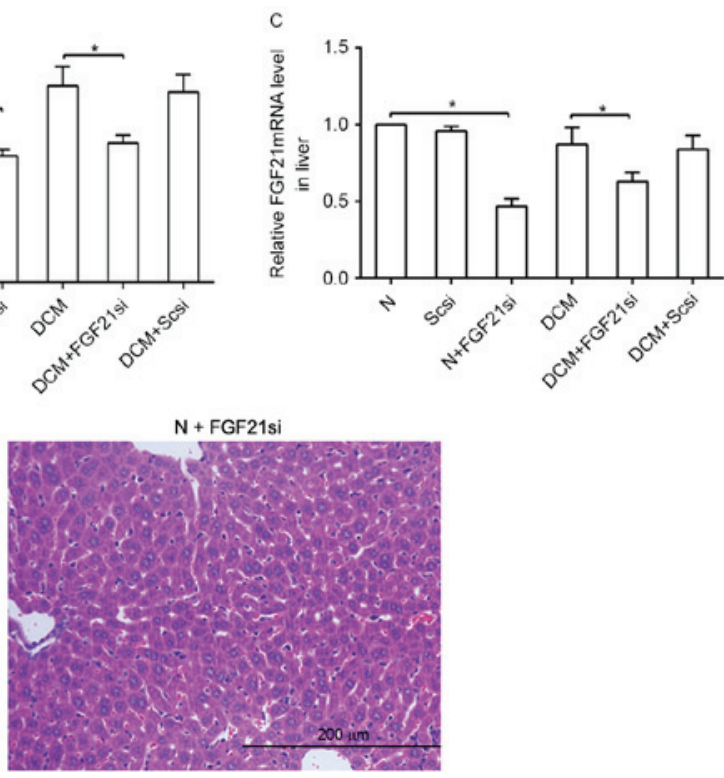

Figure 1. FGF21 concentration in mice in the different groups. (A) Plasma FGF21 concentration determined by ELISA. (B) Cardiac and (C) liver FGF21 mRNA expression levels were analyzed by reverse transcription-quantitative polymerase chain reaction. (D) Hematoxylin and eosin staining of liver tissues obtained by mice in the normal group and normal + FGF21 siRNA groups (magnification, $\mathrm{x} 400$; scale bar, 200 $\mu$ m). * $<0.05$. FGF21, fibroblast growth factor 21; N, normal; DCM, diabetic cardiomyopathy; Scsi, scrambled siRNA; FGF21si, FGF21 siRNA.

Notably, the DCM + FGF21 siRNA group demonstrated a markedly higher blood glucose level $(\mathrm{P}<0.05)$ compared with the DCM and DCM + scrambled siRNA groups, indicating that FGF21 inhibition promoted an increase in the blood glucose level of DCM mice. However, no significant difference was identified between the normal, normal + scrambled siRNA and normal + FGF21 siRNA groups in terms of the body weight and blood glucose $(\mathrm{P}>0.05)$, indicating that the regulating effect of FGF21 only occurred in DCM mice.

FGF21 siRNA inhibits the FGF21 expression. The plasma, cardiac and liver FGF21 expression levels were evaluated to assess the effectiveness of FGF21 siRNA (Fig. 1). The liver tissue in the normal and FGF21 siRNA groups were compared to reveal if FGF21 siRNA itself had any significant effect on the liver. A significant difference in FGF21 expression levels was observed between the normal and normal + FGF21 siRNA groups, as well as between the DCM and DCM + FGF21 siRNA group, indicated that FGF21 inhibition was successfully performed. By contrast, a non-significant difference was observed between the normal + scrambled siRNA and normal groups, as well as between the DCM + scrambled siRNA and DCM groups, suggesting that the scrambled siRNA was not able to markedly affect the FGF21 expression and indicating the specific effect of FGF21 siRNA on mice.

Inhibition of FGF21 aggravates the diabetes-induced cardiac hypertrophy and dysfunction. The cardiac structure and function were evaluated by TTE (Table III). Structural indicators of DCM group were significantly increased in comparison with those of the normal group, including the LV mass, LVIDd, LVIDs, IVSd, IVSs, PWTd and PWTs, while functional indicators of the DCM group, including the EF and FS, were significantly lower as compared with those of the normal group. 
Table III. Cardiac structure and function of mice in each group.

\begin{tabular}{lcccrrr}
\hline Parameter & Normal & Normal + Scsi & Normal + FGF21si & DCM & DCM + Scsi & DCM + FGF21si \\
\hline EF (\%) & $92.44 \pm 1.43$ & $90.30 \pm 1.26$ & $89.03 \pm 1.97$ & $82.20 \pm 3.21^{\mathrm{a}}$ & $81.6 \pm 2.47$ & $71.45 \pm 3.62^{\mathrm{b}}$ \\
FS (\%) & $65.67 \pm 2.74$ & $64.20 \pm 2.3$ & $62.71 \pm 2.15$ & $50.98 \pm 2.67^{\mathrm{a}}$ & $49.89 \pm 2.31$ & $40.99 \pm 2.27^{\mathrm{b}}$ \\
LV mass (mg) & $46.28 \pm 8.29$ & $54.30 \pm 7.69$ & $61.28 \pm 3.02$ & $72.38 \pm 1.37^{\mathrm{a}}$ & $71.87 \pm 1.25$ & $81.90 \pm 3.93^{\mathrm{b}}$ \\
LVIDd (mm) & $2.21 \pm 0.15$ & $2.37 \pm 0.13$ & $2.48 \pm 0.07$ & $2.90 \pm 0.10^{\mathrm{a}}$ & $2.86 \pm 0.12$ & $3.15 \pm 0.12^{\mathrm{b}}$ \\
LVIDs (mm) & $0.99 \pm 0.06$ & $0.98 \pm 0.06$ & $1.09 \pm 0.05$ & $1.48 \pm 0.16^{\mathrm{a}}$ & $1.48 \pm 0.13$ & $1.82 \pm 0.06^{\mathrm{b}}$ \\
IVSd (mm) & $0.75 \pm 0.07$ & $0.748 \pm 0.04$ & $0.84 \pm 0.03$ & $0.93 \pm 0.03^{\mathrm{a}}$ & $0.937 \pm 0.02$ & $1.09 \pm 0.08^{\mathrm{b}}$ \\
IVSs (mm) & $1.28 \pm 0.04$ & $1.31 \pm 0.03$ & $1.36 \pm 0.09$ & $1.55 \pm 0.03^{\mathrm{a}}$ & $1.52 \pm 0.04$ & $1.66 \pm 0.02^{\mathrm{b}}$ \\
PWTd (mm) & $0.80 \pm 0.11$ & $0.83 \pm 0.07$ & $0.87 \pm 0.02$ & $0.94 \pm 0.04^{\mathrm{a}}$ & $0.96 \pm 0.03$ & $1.07 \pm 0.046^{\mathrm{b}}$ \\
PWTs (mm) & $1.09 \pm 0.11$ & $1.10 \pm 0.09$ & $1.14 \pm 0.13$ & $1.45 \pm 0.06^{\mathrm{a}}$ & $1.42 \pm 0.05$ & $1.64 \pm 0.06^{\mathrm{b}}$
\end{tabular}

${ }^{\mathrm{a}} \mathrm{P}<0.05$ vs. $\mathrm{N}$ group; ${ }^{\mathrm{b}} \mathrm{P}<0.05$ vs. DCM group. Data were analyzed by one-way analysis of variance, followed by a post hoc Turkey's test. Values are reported as the mean \pm standard deviation. EF, ejection fraction; FS, fractional shortening; LV, left ventricular; LVIDd, LV internal diameter during diastole; LVIDs, LV internal diameter during systole; IVSd, interventricular septum during diastole; IVSs, interventricular septum during systole; PWTd, posterior wall thickness during diastole; PWTs, posterior wall thickness during systole; DCM, diabetic cardiomyopathy; Scsi, scrambled siRNA; FGF21si, FGF21 siRNA; FGF21, fibroblast growth factor 21.

A

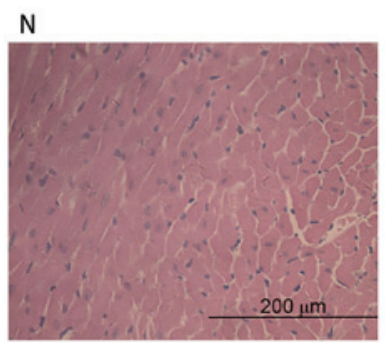

DCM

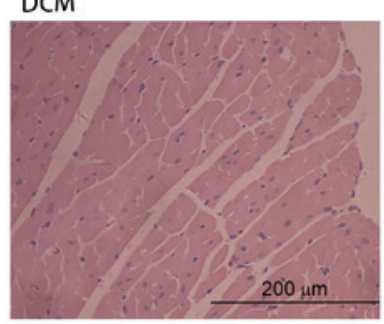

B

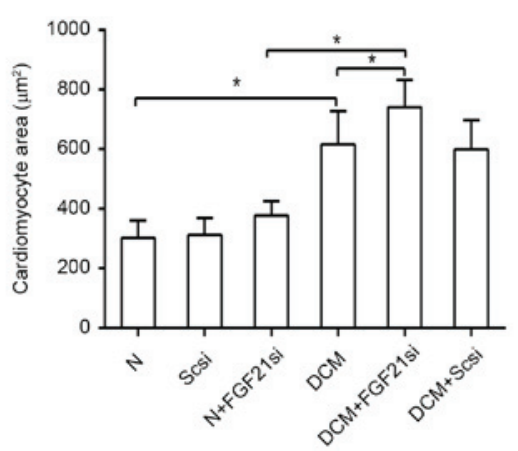

Scsi

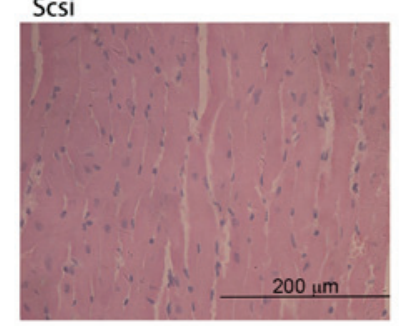

DCM+FGF21si

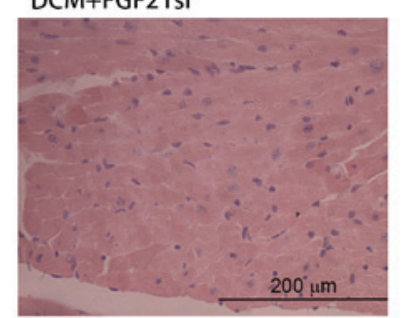

$\mathrm{N}+\mathrm{FGFsi}$

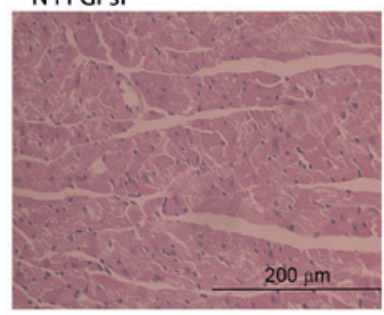

DCM+Scsi

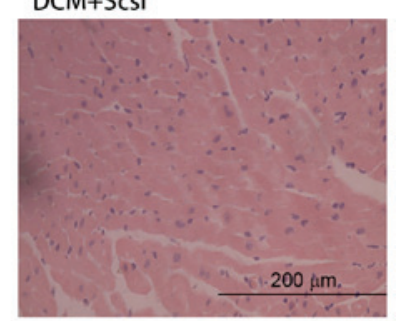

C

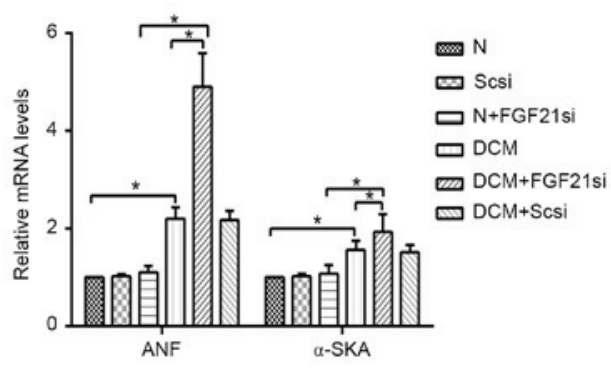

Figure 2. Morphology of cardiomyocytes and expression of hypertrophic markers. (A) Hematoxylin and eosin staining of cardiac tissue (magnification, x400; scale bar, $200 \mu \mathrm{m}$ ). (B) Quantification of cardiomyocyte cross-section area. (C) Relative mRNA expression levels of hypertrophic markers, ANF and $\alpha$-SKA. ${ }^{*} \mathrm{P}<0.05$. ANF, atrial natriuretic peptide; $\alpha$-SKA, $\alpha$-skeletal actin; FGF21, fibroblast growth factor 21; N, normal; DCM, diabetic cardiomyopathy; Scsi, scrambled siRNA; FGF21si, FGF21 siRNA.

In addition, structural indicators in the DCM + FGF21 siRNA group were significantly enhanced compared with those in the
DCM group, accompanied by reduced functional indicators. However, no significant difference was observed between the 
A

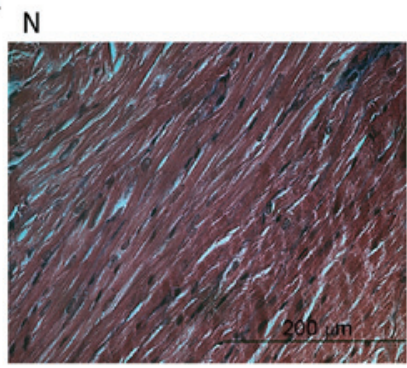

DCM

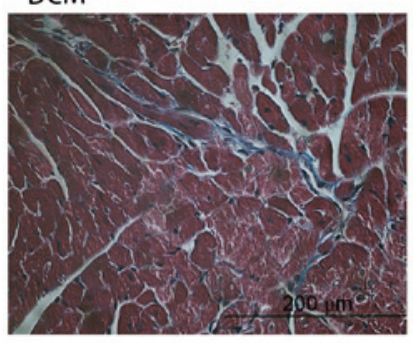

Scsi

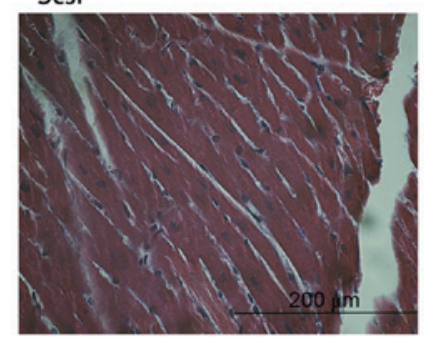

DCM+FGF21si

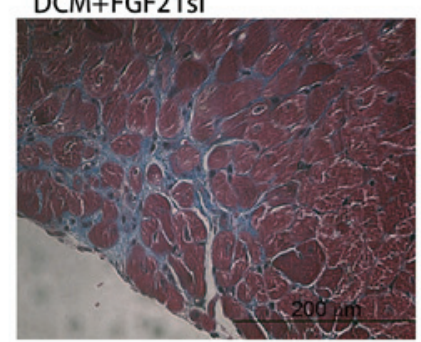

$\mathrm{N}+\mathrm{FGF} 21 \mathrm{si}$

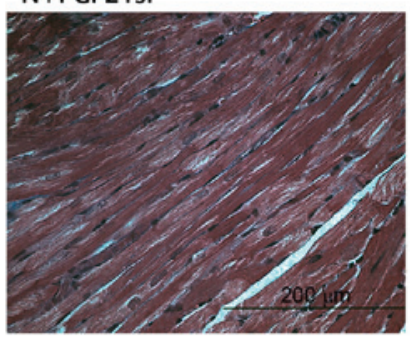

$\mathrm{DCM}+\mathrm{Scsi}$

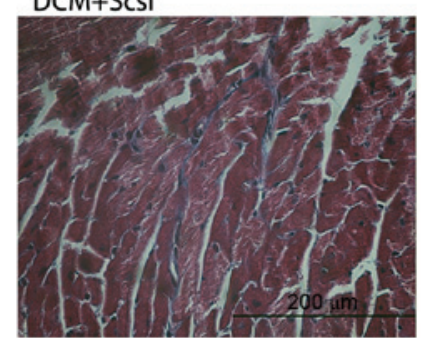

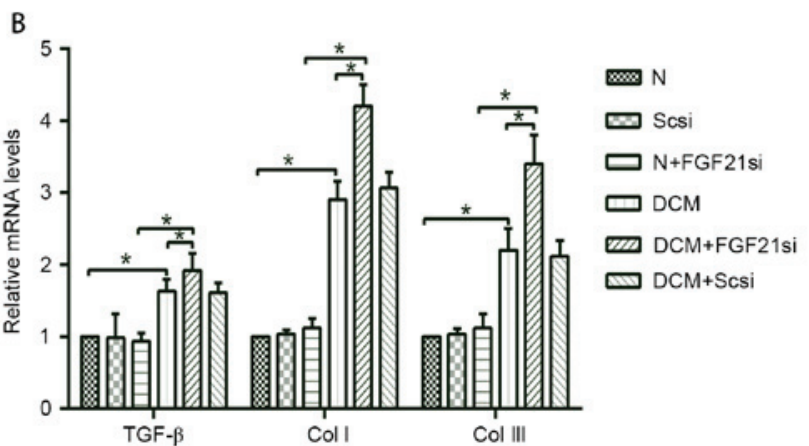

Figure 3. Cardiac fibrosis and expression levels of TGF- $\beta$, Col I and Col III. (A) Masson's trichrome staining was performed in cardiac tissues (magnification, $\mathrm{x} 400$; scale bar, $200 \mu \mathrm{m}$ ). (B) Relative mRNA expression levels of fibrosis-associated factors, including TGF- $\beta$, Col I and Col III. * $<0.05$. TGF- $\beta$, transforming growth factor- $\beta$; Col, collagen; FGF21, fibroblast growth factor 21; N, normal; DCM, diabetic cardiomyopathy; Scsi, scrambled siRNA; FGF21si, FGF21 siRNA.

normal, normal + scrambled siRNA and normal + FGF21 siRNA groups, as well as between the DCM + scrambled siRNA and DCM groups in the IVSd, IVSs, PWTd, PWTs, EF and FS values, indicating that FGF21 inhibition further thickened the cardiac wall and deteriorated the function of the heart in DCM mice.

FGF21 inhibition promotes the myocardial hypertrophy and cardiac fibrosis. The myocardial morphology (Fig. 2A) was observed under a light microscope, the cardiomyocyte areas were assessed by ImageJ software (Fig. 2B), while RT-qPCR was used to measure the expression levels of the hypertrophic markers ANF and $\alpha$-SKA (Fig. 2C). Furthermore, the cardiac fibrosis (Fig. 3A) was examined under the light microscope, and the levels of fibrosis-associated factors (TGF- $\beta$, Col I and Col III; Fig. 3B) were evaluated by RT-qPCR.

Light microscopy examination indicated apparent myocardial hypertrophy and cardiac fibrosis in the DCM and DCM + scrambled siRNA groups, which deteriorated in the DCM + FGF21 siRNA group. By contrast, no evident morphologic and fibrotic alterations were observed in the scramble siRNA and normal + FGF21 siRNA groups when compared with the normal group. The mRNA expression levels of hypertrophic markers ( $\alpha$-SKA and ANF) and fibrosis-associated factors (Col I, Col III and TGF- $\beta$ ) exhibited no significant alterations among the normal, normal + scrambled siRNA or normal + FGF21 siRNA groups, as well as between the $\mathrm{DCM}$ and DCM + scramble siRNA groups. However, these indicators were significantly increased in the DCM group when compared with the normal group. Furthermore, mRNA levels of these factors in the DCM + FGF21 siRNA group were markedly enhanced compared with the DCM or the normal + FGF21 siRNA groups. These findings indicated that FGF21 inhibition by siRNA injection aggravated the myocardial hypertrophy and cardiac fibrosis in DCM mice.

FGF21 inhibition elevates lipid accumulation. The cardiac lipid accumulation status was detected by counting the lipid droplets under a TEM (Fig. 4A and B) and assessing the triglyceride content in heart tissue (Fig. 4C). Additionally, the plasma triglyceride and cholesterol levels were assessed (Fig. 4D and $\mathrm{E}$ ). The number of lipid drops in the heart tissue of the DCM group was evidently increased in comparison with the normal group, although it was lower when compared with the DCM + FGF21 siRNA group (both $\mathrm{P}<0.05$ ). Furthermore, the cardiac triglyceride, plasma triglyceride and plasma cholesterol 
A

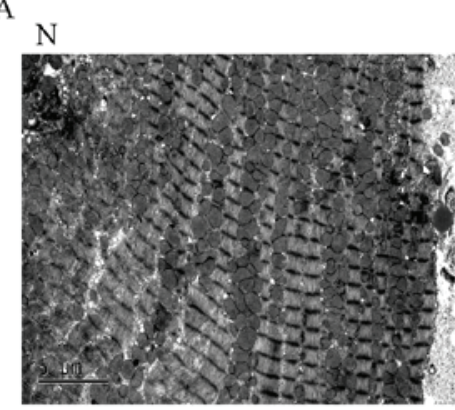

DCM

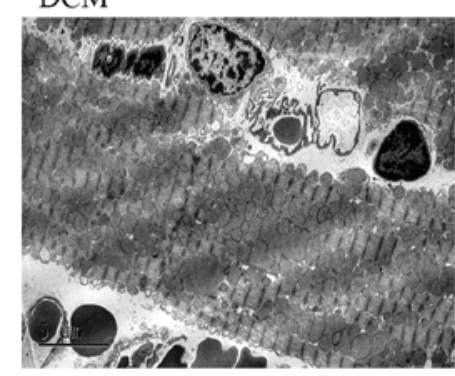

Scsi

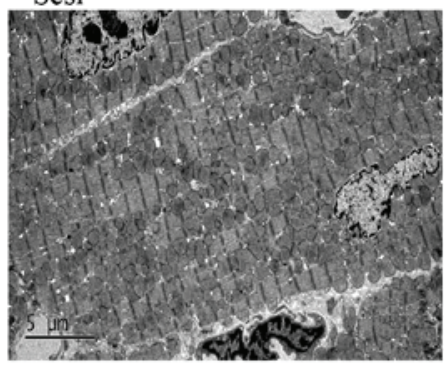

DCM+FGF21si

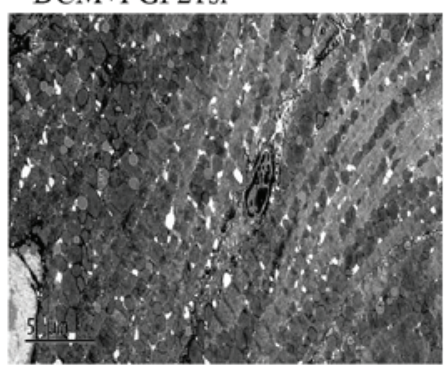

N+FGF21si

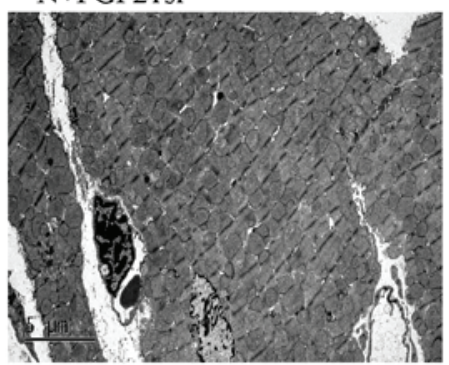

$\mathrm{DCM}+\mathrm{Scsi}$

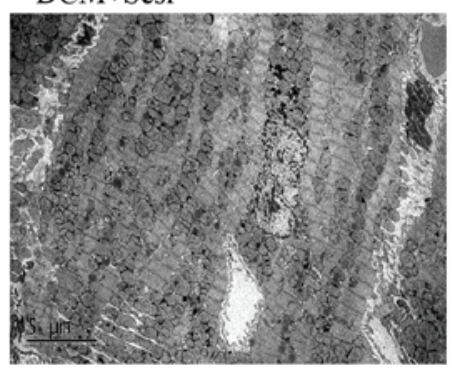

B

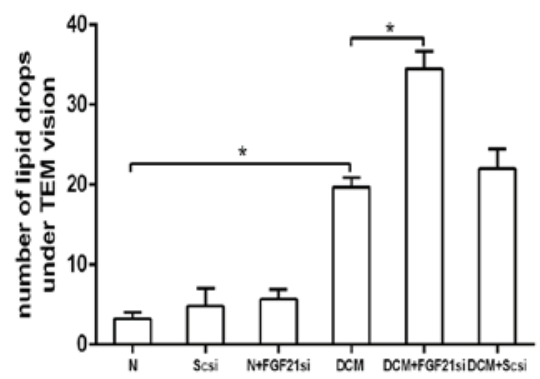

D

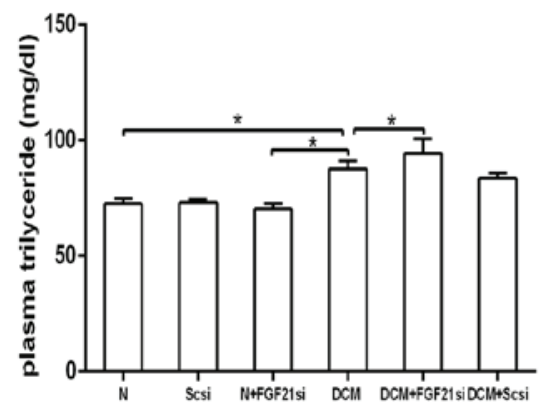

C

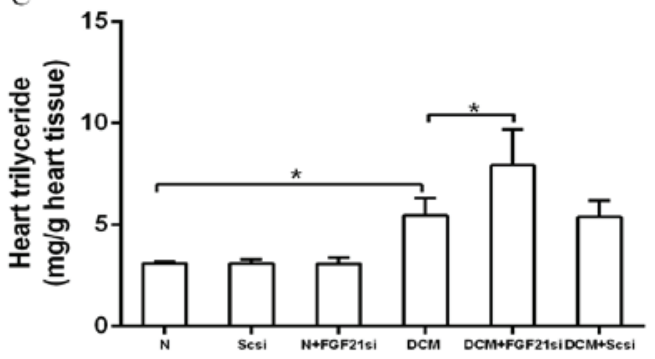

$\mathrm{E}$

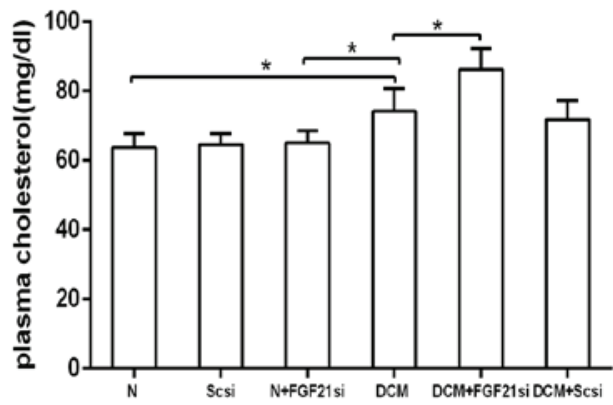

Figure 4. Quantification of lipid droplets, as well as heart triglyceride, plasma triglyceride and cholesterol concentrations in the different groups. (A) Lipid droplets observed by examination under a transmission electron microscope. Magnification, x4,200. (B) Quantitative analysis of lipid droplets. Analysis of (C) heart triglyceride, (D) plasma triglyceride and (E) plasma cholesterol levels. * $\mathrm{P}<0.05$. FGF21, fibroblast growth factor 21; N, normal; DCM, diabetic cardiomyopathy; Scsi, scrambled siRNA; FGF21si, FGF21 siRNA.

concentrations presented no significant differences among the normal, normal + scrambled siRNA and normal + FGF21 siRNA groups, as well as between the DCM + scrambled siRNA and DCM groups. By contrast, these concentrations were significantly increased in the DCM group compared with the normal group, as well as in the DCM + FGF21 siRNA group compared with the DCM and normal groups. All these observations suggested that FGF21 inhibition promoted lipid accumulation in DCM mice.

FGF21 inhibition affects the PGC-1 $\alpha$ and $C D 36$ expression levels. CD36 and PGC-1 $\alpha$ are two factors associated with lipid metabolism. As CD36 is a lipid transport protein and serves a role in mediating the cardiac fatty acid transportation and utilization (14), increased expression of this factor in the heart can lead to cardiac lipid accumulation (15). In addition, PGC-1 $\alpha$ is critical for regulating fatty acid $\beta$-oxidation and mediating the effect of FGF21 on lipid metabolism (16). Therefore, in the present study, the concentrations of PGC1- $\alpha$ and CD36 were used to estimate the status of the cardiac lipid metabolism. The protein expression levels of PGC-1 $\alpha$ and CD36 were detected by western blot analysis (Fig. 5A). PGC-1 $\alpha$ expression in the DCM group was inhibited as compared with that of the normal group, and its expression was further decreased in the 
A

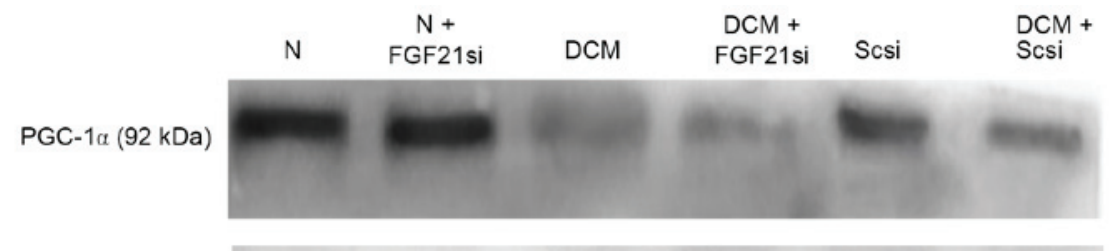

$\operatorname{CD} 36(88 \mathrm{kDa})$

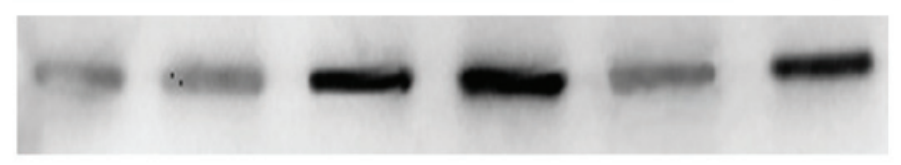

GAPDH $(36 \mathrm{kDa})$

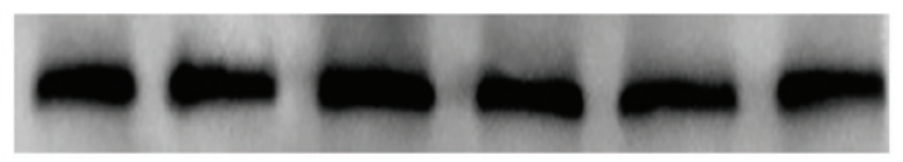

B

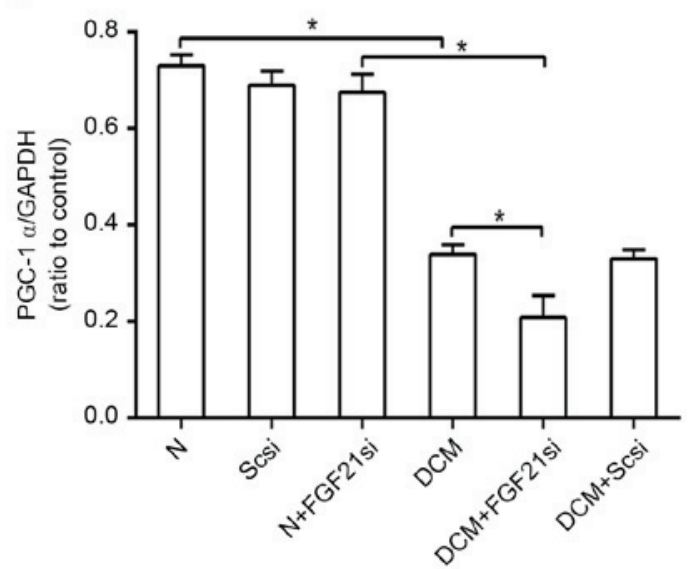

C

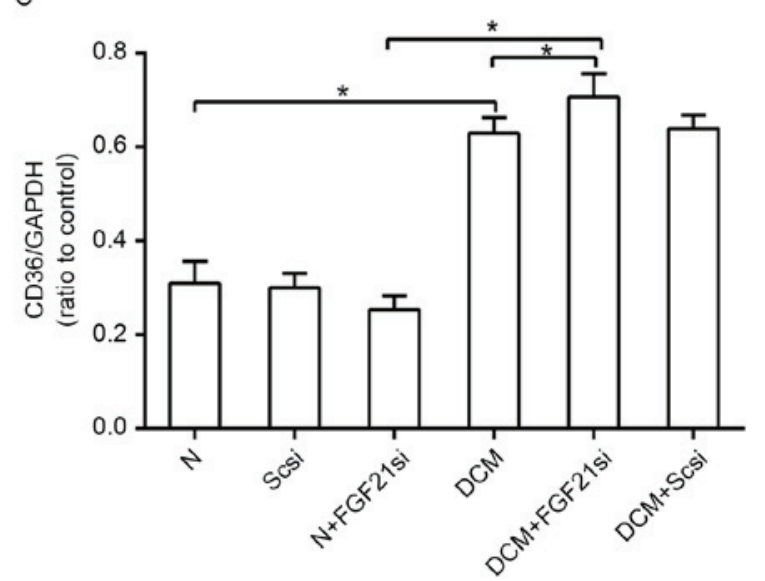

Figure 5. Inhibition of FGF21 in DCM mice resulted in reduced PGC-1 $\alpha$ and enhanced CD36 levels. (A) Western blot analysis of the expression levels of PGC-1 $\alpha$ and CD36 in the various treatment groups. The quantified levels of (B) PGC-1 $\alpha$ and (C) CD36 are also presented. "P<0.05. PGC-1 $\alpha$, peroxisome proliferator-activated receptor $\gamma$ co-activator $1 \alpha$; FGF21, fibroblast growth factor 21; N, normal; DCM, diabetic cardiomyopathy; Scsi, scrambled siRNA; FGF21si, FGF21 siRNA.

$\mathrm{DCM}+\mathrm{FGF} 21$ siRNA group when compared with the DCM and normal + FGF21 siRNA groups $(\mathrm{P}<0.05)$. No significant difference was, however, identified between the three normal groups (Fig. 5B). In contrast to the PGC-1 $\alpha$ level, the protein expression of CD36 in the DCM + FGF21 siRNA group was markedly upregulated when compared with the DCM and normal + FGF21 siRNA groups, while the expression level in the DCM group was also significantly increased in comparison with that of the normal group, while no significant difference was identified between the normal and normal + FGF21 siRNA group (Fig. 5C). All these findings indicated that absence of FGF21 decreased the expression of PGC-1 $\alpha$ and promoted the expression of CD36 in the DCM mice.

\section{Discussion}

DCM was initially defined by Rubler et al (16), reporting the cases of four diabetic patients with congestive heart failure and normal coronary arteries in 1972. DCM is a common condition in the general population, with a prevalence of $1.1 \%$ (17) characterized with cardiac structural and functional alterations, including LV hypertrophy, as well as diastolic and systolic dysfunction (18). At the later stages, systolic dysfunction characterized by the inability of LV to pump sufficient volume of blood emerges in DCM patients (18).
It have been reported that hyperglycemia and hyperlipidemia are major contributors to DCM, while FGF21 exhibited anti-hyperglycemic and anti-hyperlipidemic abilities in diabetic rodent and monkey models (19). FGF21 participates in regulating the lipid homoeostasis in adipose tissue (20) and in the liver (3). In addition, FGF21 was also reported to induce renal protection, partially by lessening the renal lipid accumulation through enhancing fatty acid oxidation and lipolysis (21). Therefore, in the current study, it was hypothesized that FGF21 inhibition may further elevate the levels of plasma glucose, and plasma and cardiac lipid accumulation, leading to the accelerated and aggravated development of DCM.

In the present study, FGF21 was effectively inhibited by FGF21 siRNA, which was confirmed by the significantly decreased FGF21 expression in normal and DCM mice treated with FGF21 siRNA. It was observed that the plasma and liver FGF21 expression levels of DCM mice were markedly reduced compared with those in normal mice, which may be caused by tissue damage at the late stages of DCM, resulting in decreased secretion of FGF21. As there was no significant change in the liver between the normal and normal + FGF21 siRNA groups, it was suggested that FGF21 siRNA alone did not cause liver dysfunction; thus it is concluded that the observations in these experiments were not a result of liver dysfunction. 
Lipid droplets store excess lipids, particularly triglycerides, when the lipid concentration surpasses the required amounts for cellular structures and ATP generation. A small number of droplets can be detected in the normal heart under basic conditions (22); however, significantly increased lipid droplets imply that the lipid metabolism is unbalanced. In the present study, a greater number of cardiac lipid droplets was identified in the hearts of DCM mice than in normal mice, while increased droplets were also observed in the DCM + FGF21 siRNA group compared with the DCM group. These data suggested that the lipid metabolism in DCM mice was unbalanced and that the unbalanced status was worse in mice of the DCM + FGF21 siRNA group. In accordance with these findings on lipid metabolism, increased levels of cardiac triglyceride, plasma triglyceride and cholesterol levels were detected in DCM mice compared with normal mice; subsequent to inhibition of FGF21 expression by FGF21 siRNA, these levels further increased. The aforementioned findings suggested that a lack of FGF21 in DCM mice caused enhanced lipid accumulation.

Increased IVSd, IVSs, PWTd, PWTs and LV mass values, along with the H\&E staining of heart tissues, demonstrated that cardiac hypertrophy existed in DCM mice in the present study. Furthermore, Masson's trichrome staining, and decreased EF and FS revealed that cardiac fibrosis and dysfunction were also present in DCM mice. Long-term cardiac remodeling is known to lead to aggravated ventricular hypertrophy and cardiac fibrosis, along with contractile dysfunction $(12,23)$. Deteriorated cardiac hypertrophy and fibrosis resulted in further inhibition of EF and FS in mice of the DCM + FGF21 siRNA group, indicating that these mice suffered more severe cardiac remodeling, which was not observed in the normal, scrambled siRNA and normal + FGF21 siRNA groups. According to the aforementioned analyses, mice in the DCM + FGF21 siRNA group, followed by the DCM mice, accumulated the highest level of triglycerides and cholesterol, suffering the highest degree of cardiac hypertrophy and fibrosis, as well as the lowest cardiac function. These results are in accordance with earlier studies reporting that excess lipid accumulation in cardiomyocytes is responsible for diabetic cardiac dysfunction (19), and that triglyceride concentration is associated with LV hypertrophy in patients with diabetes (24), suggesting the association of the lipid accumulation observed in the present study with cardiac remodeling in DCM.

$\mathrm{CD} 36$, as a lipid transport protein, has been reported to mediate cardiac fatty acid transport and utilization (14). It was initially described as a component of the platelet membrane and later identified as a receptor for thrombospondin-1 and a class B scavenger receptor B, which is associated with the binding of modified and native lipoproteins and anionic phospholipids (25-27). In murine diabetic models, as well as in mice fed a high-fat diet, increased CD36 expression in the heart mediated excess fatty acid uptake, which led to cardiac lipid accumulation, and this expression was associated with the parenchymal cell lipid metabolism (15). CD36 deletion was also reported to improve the heart function in aged mice that were fed with a diet enriched in medium chain fatty acids (28). In the present study, CD36 expression was enhanced in the DCM group in comparison with the normal group, and even higher expression was observed in the DCM + FGF21 siRNA group compared with the DCM group. Considering that more lipids were accumulated in DCM mice compared with normal mice and in mice of the DCM + FGF21 siRNA group compared with DCM mice, it is concluded that FGF21 inhibition regulated the CD36 upregulation, resulting in increased lipid concentration and partly contributing to the significantly elevated lipid accumulation.

PGC- $1 \alpha$ is a transcriptional coactivator protein and its expression can be induced by the nutritional status and stimuli, such as cold and exercise (29-32). PGC-1 $\alpha$ serves a critical role in regulating the fatty acid $\beta$-oxidation and mediating the effect of FGF21 on lipid metabolism (33). In the present study, PGC-1 $\alpha$ expressed in DCM mice was downregulated, accompanied by increased lipid accumulation. Its expression in mice of the DCM + FGF21 siRNA group was further decreased, along with the significant increase in accumulated lipids. These findings demonstrated that FGF21 regulated PGC-1 $\alpha$ expression, contributing to lipid accumulation. Thus, the current study revealed that FGF21 inhibition induced PGC- $1 \alpha$ expression downregulation in DCM mice, contributing to the significantly elevated lipid concentration along with the CD36 expression increase.

In conclusion, the present study observed that FGF21 inhibition caused more significant hyperglycemia and hyperlipidemia in DCM mice. Furthermore, FGF21 inhibition upregulated CD36 expression and downregulated PGC- $1 \alpha$ expression, causing excess lipid uptake and leading to increased lipid accumulation, which promoted cardiac remodeling involving cardiac hypertrophy, cardiac fibrosis and cardiac dysfunction, further accelerating the development of DCM. Therefore, it is suggested that FGF21 may be a potential tool in the development of medical strategies for the prevention of DCM.

\section{References}

1. Lorenzo-Almorós A, Tuñón J, Orejas M, Cortés M, Egido J and Lorenzo O: Diagnostic approaches for diabetic cardiomyopathy. Cardiovasc Diabetol 16: 28, 2017.

2. Wang J, Song Y, Wang Q, Kralik PM and Epstein PN: Causes and characteristics of diabetic cardiomyopathy. Rev Diabet Stud 3: 108-117, 2006.

3. Badman MK, Pissios P, Kennedy AR, Koukos G, Flier JS and Maratos-Flier E: Hepatic fibroblast growth factor 21 is regulated by PPARalpha and is a key mediator of hepatic lipid metabolism in ketotic states. Cell Metab 5: 426-437, 2007.

4. Galman C, Lundåsen T, Kharitonenkov A, Bina HA, Eriksson M, Hafström I, Dahlin M, Amark P, Angelin B and Rudling M: The circulating metabolic regulator FGF21 is induced by prolonged fasting and PPARalpha activation in man. Cell Metab 8: 169-174, 2008.

5. Cheng P, Zhang F, Yu L, Lin X, He L, Li X, Lu X, Yan X, Tan Y and Zhang C: Physiological and pharmacological roles of FGF21 in cardiovascular diseases. J Diabetes Res 2016: 1540267, 2016.

6. Planavila A, Redondo I, Hondares E, Vinciguerra M, Munts C, Iglesias R, Gabrielli LA, Sitges M, Giralt M, van Bilsen M and Villarroya F: Fibroblast growth factor 21 protects against cardiac hypertrophy in mice. Nat Commun 4: 2019, 2013.

7. Chow WS, Xu A, Woo YC, Tso AW, Cheung SC, Fong $\mathrm{CH}$, Tse HF, Chau MT, Cheung BM and Lam KS: Serum fibroblast growth factor-21 levels are associated with carotid atherosclerosis independent of established cardiovascular risk factors. Arterioscler Thromb Vasc Biol 33: 2454-2459, 2013.

8. Semba RD, Crasto C, Strait J, Sun K, Schaumberg DA and Ferrucci L: Elevated serum fibroblast growth factor 21 is associated with hypertension in community-dwelling adults. J Hum Hypertens 27: 397-399, 2013.

9. Lin Z, Wu Z, Yin X, Liu Y, Yan X, Lin S, Xiao J, Wang X, Feng $\mathrm{W}$ and Li X: Serum levels of FGF-21 are increased in coronary heart disease patients and are independently associated with adverse lipid profile. PLoS One 5: e15534, 2010. 
10. Planavila A, Redondo-Angulo I, Ribas F, Garrabou G Casademont J, Giralt $\mathrm{M}$ and Villarroya F: Fibroblast growth factor 21 protects the heart from oxidative stress. Cardiovasc Res 106: 19-31, 2015.

11. Wang R, Yi X, Li X and Jiang X: Fibroblast growth factor-21 is positively associated with atrial fibrosis in atrial fibrillation patients with rheumatic heart disease. Int J Clin Exp Pathol 8: 14901-14908, 2015.

12. Zhang W, Chu S, Ding W and Wang F: Serum level of fibroblast growth factor 21 is independently associated with acute myocardial infarction. PLoS One 10: e0129791, 2015.

13. Livak KJ and Schmittgen TD: Analysis of relative gene expression data using real-time quantitative PCR and the 2(-Delta Delta C(T)) method. Methods 25: 402-408, 2001.

14. Koonen DP, Glatz JF, Bonen A and Luiken JJ: Long-chain fatty acid uptake and FAT/CD36 translocation in heart and skeletal muscle. Biochim Biophys Acta 1736: 163-180, 2005.

15. Greenwalt DE, Scheck SH and Rhinehart-Jones T: Heart CD36 expression is increased in murine models of diabetes and in mice fed a high fat diet. J Clin Invest 96: 1382-1388, 1995.

16. Rubler S, Dlugash J, Yuceoglu YZ, Kumral T, Branwood AW and Grishman A: New type of cardiomyopathy associated with diabetic glomerulosclerosis. Am J Cardiol 30: 595-602, 1972.

17. Dandamudi S, Slusser J, Mahoney DW, Redfield MM, Rodeheffer RJ and Chen HH: The prevalence of diabetic cardiomyopathy: A population-based study in Olmsted County, Minnesota. J Card Fail 20: 304-309, 2014.

18. Yilmaz S, Canpolat U, Aydogdu S and Abboud HE: Diabetic cardiomyopathy; summary of 41 years. Korean Circ J 45: 266-272, 2015

19. Boudina S and Abel ED: Diabetic cardiomyopathy revisited. Circulation 115: 3213-3223, 2007.

20. Muise ES, Souza S, Chi A, Tan Y, Zhao X, Liu F, Dallas-Yang Q, Wu M, Sarr T, Zhu L, et al: Downstream signaling pathways in mouse adipose tissues following acute in vivo administration of fibroblast growth factor 21. PLoS One 8: e73011, 2013.

21. Zhang C, Shao M, Yang H, Chen L, Yu L, Cong W, Tian H, Zhang F, Cheng P, Jin L, et al: Attenuation of hyperlipidemiaand diabetes-induced early-stage apoptosis and late-stage renal dysfunction via administration of fibroblast growth factor-21 is associated with suppression of renal inflammation. PLoS One 8: e82275, 2013.

22. Goldberg IJ, Trent CM and Schulze PC: Lipid metabolism and toxicity in the heart. Cell Metab 15: 805-812, 2012.

23. Neely JR, Rovetto MJ and Oram JF: Myocardial utilization of carbohydrate and lipids. Prog Cardiovasc Dis 15: 289-329, 1972.
24. Herrero P, Peterson LR, McGill JB, Matthew S, Lesniak D, Dence $\mathrm{C}$ and Gropler RJ: Increased myocardial fatty acid metabolism in patients with type 1 diabetes mellitus. J Am Coll Cardiol 47: 598-604, 2006.

25. Asch AS, Barnwell J, Silverstein RL and Nachman RL: Isolation of the thrombospondin membrane receptor. J Clin Invest 79: 1054-1061, 1987.

26. Silverstein RL, Asch AS and Nachman RL: Glycoprotein IV mediates thrombospondin-dependent platelet-monocyte and platelet-U937 cell adhesion. J Clin Invest 84: 546-552, 1989.

27. Endemann G, Stanton LW, Madden KS, Bryant CM, White RT and Protter AA: CD36 is a receptor for oxidized low density lipoprotein. J Biol Chem 268: 11811-11816, 1993.

28. Koonen DP, Febbraio M, Bonnet S, Nagendran J, Young ME, Michelakis ED and Dyck JR: CD36 expression contributes to age-induced cardiomyopathy in mice. Circulation 116: 2139-2147, 2007.

29. Puigserver P, Rhee J, Donovan J, Walkey CJ, Yoon JC, Oriente F, Kitamura Y, Altomonte J, Dong H, Accili D and Spiegelman BM: Insulin-regulated hepatic gluconeogenesis through FOXO1-PGC-1alpha interaction. Nature 423: 550-555, 2003.

30. Lin J, Handschin C and Spiegelman BM: Metabolic control through the PGC-1 family of transcription coactivators. Cell Metab 1: 361-370, 2005.

31. Finck BN and Kelly DP: PGC-1 coactivators: Inducible regulators of energy metabolism in health and disease. J Clin Invest 116: 615-622, 2006

32. Handschin $\mathrm{C}$ and Spiegelman BM: Peroxisome proliferator-activated receptor gamma coactivator 1 coactivators, energy homeostasis, and metabolism. Endocr Rev 27: 728-735, 2006.

33. Potthoff MJ, Inagaki T, Satapati S, Ding X, He T, Goetz R, Mohammadi M, Finck BN, Mangelsdorf DJ, Kliewer SA and Burgess SC: FGF21 induces PGC-1alpha and regulates carbohydrate and fatty acid metabolism during the adaptive starvation response. Proc Natl Acad Sci USA 106: 10853-10858, 2009.

(i) $($ This work is licensed under a Creative Commons Attribution-NonCommercial-NoDerivatives 4.0 International (CC BY-NC-ND 4.0) License. 